\title{
Multiple Sclerosis Patients in Saudi Arabia: Prevalence of Depression and its Extent of Severity
}

\author{
Hamad Alhussain ${ }^{1}$, Abdulaziz A. Aldayel ${ }^{1}$, Abdulaziz Alenazi ${ }^{1}$, Faris Alowain ${ }^{1}$ \\ 1. Medicine, Imam Mohammad Ibn Saud Islamic University, Riyadh, SAU
}

Corresponding author: Abdulaziz A. Aldayel, aldaye19@gmail.com

\section{Abstract}

\section{Background}

Multiple sclerosis (MS) is a serious chronic autoimmune disorder of the central nervous system of unknown etiology. MS-related depression is a common mood disorder recognized within the medical community. However, their association is ambiguous, underdiagnosed, undertreated and less reported.

\section{Objectives}

The study aimed to estimate the point prevalence and severity of depression among multiple sclerosis patients in Saudi Arabia.

\section{Materials and methods}

We conducted an observational cross-sectional study among multiple sclerosis patients in Riyadh region, Saudi Arabia. Patients filled demographic data and Patient Health Questionnaire-9 (PHQ-9) to determine depression. Those who did not meet the age, disease duration, and regular follow-up eligibility criteria were excluded from the study.

\section{Results}

We enrolled 238 MS patients in the study, male patients represented 39.1\% ( $n=93)$ while females accounted for $60.9 \%(n=145)$ (male:female ratio 0.64$)$. The mean age of the study population was $32.07 \pm 7.93$ years. The mean duration of the disease was $7.06 \pm 4.7$ years. We determined that $89.9 \%(n=214)$ of the patients showed mild to severe depression symptoms ( $55.46 \%$ of the females, and $34.4 \%$ of the males; $\mathrm{p}=0.474)$. We further found that $37.39 \%(n=89)$ and $65.13 \%(n=155)$ of the depressed patients were unemployed $(p=$ $0.039)$ and were non-smokers $(p=0.097)$ respectively. Furthermore, depression severity is significantly associated with education $(\mathrm{p}=0.005)$.

Received 01/30/2020 Review began 01/31/2020 Review ended 02/10/2020 Published 02/15/2020

(c) Copyright 2020 Alhussain et al. This is an open access article distributed under the terms of the Creative Commons Attribution License CC-BY 4.0., which permits unrestricted use, distribution, and reproduction in any medium, provided the original author and source are credited.

\section{Conclusion}

High levels of depression symptoms were found among MS patients in Saudi Arabia. The relationship between MS and psychiatric conditions exists despite the uncertainty of its pathogenesis. Further longitudinal studies should be carried out to obtain more valid outcomes. Neurologists treating MS patients can play a role in studies related to the condition by investigating depressive symptoms actively and providing the data.

Categories: Neurology, Psychiatry

Keywords: multiple sclerosis, severity, depression, saudi arabia

\section{Introduction}

Over the last decades, the global interest in the burden of different chronic diseases and depression and the relationship between them has been increasing dramatically [1,2]. Multiple sclerosis (MS), one of the chronic disorders, is an autoimmune disorder of the central nervous system (CNS) of unknown etiology. The condition is characterized by demyelination and variable degrees of axonal loss that leads to significant neurological disability [3,4]. The pathogenesis of MS involves immune response directed against CNS antigens mediated through activated CD4+ myelin-reactive T cells and the possible involvement of B cells [3]. This immunopathogenesis possibly has a persistent peripheral activation of autoreactive T cells from the breakdown of self-tolerance of myelin and other CNS antigens [5]. Self-tolerance breach can be triggered in an individual with genetic susceptibility due to low vitamin D levels and environmental antigens, presumptively infection with Epstein Barr virus (EBV) [3,5]. Also, systematic reviews and meta-analysis studies comprehensively established the association between smoking habits and dosage of smoking as potential risk factors for MS development and progression [6,7]. MS is considered as one of the most common neurological disorders and causes of disability in young adults [8]. Globally, the prevalence of MS 
in 2008 was 2.1 million with a median of 30 cases per 100,000. The figure increased to 2.3 million with a median of 33 cases per 100,000 in 2013 [8]. The prevalence of MS differs with geographical variation and ethnicity. The median prevalence of MS per 100,000 is 80 in Europe, 14.9 in Eastern Mediterranean, 8.3 in the Americas, 5 in Western Pacific, 2.8 in South-East Asia, and 0.3 in Africa. The median estimated prevalence of MS in Saudi Arabia is 20.01 to 60 people per 100,000 [8]. The global average age of onset of MS symptoms is 29.2 years, with an interquartile range between 25.3 and 31.8 years, while the global male:female ratio of MS is 0.5 (two women for every single man) [8]. The concept of depression has developed from lowness of spirits of persons suffering under disease to major depressive disorder (MDD) or depression within the framework of mood disorders [9,10]. MDD is characterized by a depressed mood and diminished interest or pleasure in almost all activities throughout the day for at least two weeks [9]. The pathophysiology of depression remains uncertain but some researchers hypothesized several theories to explain the depression mechanism. It is believed that low levels of brain-derived neurotrophic factor (BDNF) leads to depression according to the neurotrophin hypothesis, but evidence of the relationship is still debatable [11]. The monoamines hypothesis provides stronger evidence by showing that the underlying pathophysiological origin of depression is due to low concentrations and depletion of serotonin, norepinephrine, and dopamine in the CNS [12]. The pathogenesis of depression in MS remains vague. Regarding epidemiology, prevalence, age of onset, and the male:female ratio of depression, they differ across the world populations [13]. The global lifetime prevalence in 2015 was estimated around 322 million or $4.4 \%$ of the world population (World Health Organization, WHO), which was $18.4 \%$ more compared to the 2005 statistics [1]. Depression manifests more in females compared to males (male:female ratio 1.6) (WHO) [13]. Depression has two peaks for age of onset, the first peak is in older adulthood ranging from 55 to 74 years (WHO), with diversity in determining the second peak as in childhood and adolescents below the age of 15 years (WHO) and to be in late 40s for men and middle 30s for women [14]. The evidence of MDD epidemiology in Saudi Arabia is lacking but the estimated prevalence is consistent with that of the Middle East and Africa at 6.6\% [15]. MDD is the third commonest health problem that causes disability in Saudi Arabia [2]. In 2018, depression was named the leading cause of world disability (WHO) and is expected to be the major contributor to disease burden by 2030 (WHO, 2008) [16]. Depression has an influence on the general health and quality of life (QOL) [2]. Risk of suicide differs significantly among depressed and normal individuals, between $2 \%$ to $7 \%$ of depressed adults are having risk to commit suicide. While normal population range is estimated at $0.5 \%$ to $1.4 \%$ or approximately 12 per 100,000 individuals per year. Several studies have attempted to address the correlation between MS and depression. In Norway, the prevalence of depression symptoms among MS patients is significantly higher than the general population at $31.4 \%$. Accordingly, $16 \%$ of the depressed untreated patients articulated the need for antidepressant treatment [17]. Another study that aimed to determine the lifetime risk of depression in a representative group of MS patients in North America reported similar results. The study concluded that $34.4 \%$ had a current or lifetime diagnosis of depression [18]. Moreover, other studies have been done to estimate the prevalence of depression symptoms in MS patients in Tunisia and Iran and they reported a prevalence of $65 \%$ and $59.4 \%$, respectively $[19,20]$. There is limited current literature about the relationship between depression and multiple sclerosis in the Saudi community. This study is meant to address the point prevalence of depression and its severity among multiple sclerosis patients in Saudi Arabia.

\section{Materials And Methods Study design and subjects}

This is an observational cross-sectional study conducted to assess depression point prevalence and its severity among multiple sclerosis patients in Saudi Arabia. The recruited sample is Saudi MS patients in Riyadh region, Saudi Arabia. Snowball sampling as designated by Goodman was used to recruit the participants online [21]. Those who have depression preceding the diagnosis of MS, with less than one year of MS symptomatology, aged less than 15 years and older than 50 years, and have no regular follow-up with a neurologist were excluded from the study.

\section{Data collection tool and research instrument}

Data collection was done through a self-administered anonymous online questionnaire that was divided into two parts. The first section concentrated on socio-demographic data of the participants, including age, gender, education level, marital status, and disease duration. The second part aimed to measure the point prevalence and severity of depression using the Patient Health Questionnaire-9 (PHQ-9). We used the standardized, valid, and reliable Arabic version of the instrument for depression screening and diagnosis consisting of nine statements based on DSM-IV [22]. The statements are set as 4-point Likert scale items and graded from 0 to 3 (not at all, several days, more than half the days, and nearly every day) to determine behavior in the past two weeks. Each application was organized and given a score between 1 to 27 as follows: 0-4 no or minimal depression, 5-9 mild depression, 10-14 moderate depression, 15-19 moderately severe depression and $\geqslant 20$ is severe depression $[22,23]$. An additional conclusive statement is included at the end of the diagnostic tool as a severity measure asserting, "How difficult have these problems made it for you to do your work, take care of things at home, or get along with other people?” The expected answers were not difficult at all, somewhat difficult, very difficult and extremely difficult [23].

\section{Data analysis}




\section{Cureus}

Data were coded and entered into the computer, Microsoft Excel Software and analyzed using SPSS version 25.0 (IBM Corp., Armonk, NY). Descriptive statistics were expressed as counts (percentage) for categorical data while mean \pm SD was used for the count data. Chi-Square test of independence was used to determine $\mathrm{P}$-values of the association between the presence of depression and sociodemographic factors as well as the association between depression severity and sociodemographic factors.

\section{Ethical approval}

All participants were informed of the objectives of the study and they participated voluntarily. A bilingual informed consent in the local language (Arabic) and English was embedded in the online data collection tool. Institutional Review Board (IRB) approval was obtained to ensure the research was conducted according to the Declaration of Helsinki. Participant anonymity was guaranteed by assigning each respondent a code number for analysis.

\section{Results}

\section{Sociodemographic data}

After application of the inclusion and exclusion criteria, a total of 238 Saudi MS patients were enrolled in the current study. The male population represented 39.1\% $(n=93)$ while the female one was $60.9 \%(n=145)$ (male:female ratio was 0.64 ). The mean age of the study population was $32.07 \pm 7.93$ years while the mean duration of the disease was $7.06 \pm 4.7$ years. Most of the participants were inhabitants of urban areas (81.5\%), had a bachelor's degree (61.8\%), employed (42.4\%) and never smoked a cigarette (74.4\%). The average household income was almost equally dispersed for each stratum as most respondents earned less than 5000 Saudi Riyal (22.7\%). Other sociodemographic characteristics are detailed in Table 1. 


\section{Cureus}

\begin{tabular}{|c|c|}
\hline Sociodemographic characteristics & $n(\%)$ \\
\hline \multicolumn{2}{|l|}{ Gender } \\
\hline Male & $93(39.1)$ \\
\hline Female & $145(60.9)$ \\
\hline Age, years ( \pm SD; median) & $32.07 \pm 7.93 ; 32.0$ \\
\hline \multicolumn{2}{|l|}{ Marital Status } \\
\hline Single & $111(46.6)$ \\
\hline Married & $107(45)$ \\
\hline Divorced & $17(7.1)$ \\
\hline Widowed & $3(1.3)$ \\
\hline \multicolumn{2}{|l|}{ Residency } \\
\hline Urban & $194(81.5)$ \\
\hline Rural & $44(18.5)$ \\
\hline \multicolumn{2}{|l|}{ Education } \\
\hline Primary school & $2(0.8)$ \\
\hline Intermediate school & $6(2.5)$ \\
\hline Secondary school & $61(25.6)$ \\
\hline University or college & $147(61.8)$ \\
\hline Postgraduate & $22(9.2)$ \\
\hline \multicolumn{2}{|l|}{ Household Income } \\
\hline Less than 5000 Saudi Riyal & $54(22.7)$ \\
\hline 5000 - 10000 Saudi Riyal & $47(19.7)$ \\
\hline 10001 - 15000 Saudi Riyal & $52(21.8)$ \\
\hline 15001 - 20000 Saudi Riyal & $35(14.7)$ \\
\hline 20001 - 25000 Saudi Riyal & $16(6.7)$ \\
\hline More than 25000 Saudi Riyal & $34(14.3)$ \\
\hline \multicolumn{2}{|l|}{ Employment Status } \\
\hline Employed & $101(42.4)$ \\
\hline Unemployed/housewife & $95(39.9)$ \\
\hline Student & $42(17.6)$ \\
\hline Duration of the Disease, years $( \pm \mathrm{SD})$; median & $7.06 \pm 4.7 ; 7.0$ \\
\hline \multicolumn{2}{|l|}{ Smoking Status } \\
\hline Heavy smoker & $37(15.5)$ \\
\hline Social smoker & $24(10.1)$ \\
\hline Not a smoker & 177 (74.4) \\
\hline
\end{tabular}

TABLE 1: Demographic data of the participants

Depression scores 


\section{Cureus}

Based on the total score $>4$ cut-off point of PHQ-9, a total of $89.9 \%(n=214)$ of the patients showed depression symptoms albeit varying from mild to severe stages (55.46\% of the females, and $34.4 \%$ of the males; $\mathrm{p}=0.474)$, while the remaining $10.1 \%(\mathrm{n}=24)$ did not show depression symptoms. Prevalence of different stages of depression is evenly distributed but the most common was mild (score 5-9) representing $24.8 \%$ followed by moderate $23.9 \%$, moderately severe $22.3 \%$, and severe $18.9 \%$. In terms of

sociodemographic factors associated with depression after using Chi-Square test of independence, a total of $37.39 \%(n=89)$ of the depressed patients were unemployed $(p=0.039)$ and $65.13 \%(n=155)$ of the depressed patients were not smokers $(p=0.097)$. Furthermore, depression severity is significantly associated with

education $(p=0.005)$. Additional information about the presence of depression, its scores, and relationship with sociodemographic factors are specified in Tables 2-5.

Presence of depression symptoms

No

Yes

Total n (\%)

$24(10.1)$

214 (89.9)

$238(100)$

\section{TABLE 2: Presence of depression}

Depression severity

None-minimal depression

Mild depression

Moderate depression

Moderately severe depression

Severe depression

Total

\section{n (\%)}

24 (10.1)

59 (24.8)

57 (23.9)

$53(22.3)$

45 (18.9)

$238(100)$

TABLE 3: Frequency distribution of depression severity 


\section{Cureus}

Sociodemographic characteristics

Gender

Male

Female

Marital Status

Single

Married

Divorced

Widowed

Residency

Urban

Rural

\section{Education}

Primary school

Intermediate school

Secondary School

University or college

Postgraduate

Household Income

Less than 5000 Saudi Riyal

5000 - 10000 Saudi Riyal

10001 - 15000 Saudi Riyal

15001 - 20000 Saudi Riyal

20001 - 25000 Saudi Riyal

More than 25000 Saudi Riyal

Employment Status

Employed

Unemployed/housewife

Student

Smoking Status

Heavy smoker

Social smoker

Not a smoker
Presence of depression symptoms

No

Yes

P-Value

11 (4.622)

82 (34.45)

0.474

$13(5.46)$

$132(55.46)$

$6(2.52)$

105 (44.12)

0.118

16 (6.72)

91 (38.24)

$2(0.84)$

15 (6.30)

$0(0)$

$3(1.26)$

21 (8.82)

173 (72.69)

0.426

3 (1.26)

41 (17.23)

$2(0.84)$

0.107

$1(0.42)$

$2(0.84)$

$5(2.10)$

59 (24.79)

16 (6.72)

131 (55.04)

17 (7.14)

4 (1.68)

50 (21.01)

0.959

6 (2.52)

41 (17.23)

5 (2.10)

47 (19.75)

4 (1.68)

31 (13.03)

$2(0.84)$

14 (5.88)

$3(1.26)$

31 (13.03)

16 (6.72)

85 (35.71)

0.039

6 (2.52)

89 (37.39)

$2(0.84)$

40 (16.81)

$2(0.84)$

35 (14.71)

0.097

TABLE 4: Presence of depression symptoms associated with sociodemographic factors

Note: Based on Chi-Square test of independence.

$0(0)$

24 (10.08)

$22(9.24)$

$155(65.13)$ 


\section{Cureus}

\begin{tabular}{|c|c|c|c|c|c|c|}
\hline \multirow{2}{*}{ Sociodemographic characteristics } & \multicolumn{5}{|c|}{ Depression severity } & \multirow{2}{*}{ P-Value } \\
\hline & None-minimal & Mild & Moderate & Moderately severe & Severe & \\
\hline \multicolumn{7}{|l|}{ Gender } \\
\hline Male & $11(4.62)$ & $22(9.24)$ & $22(9.24)$ & $18(7.56)$ & $20(8.4)$ & 0.796 \\
\hline Female & $13(5.46)$ & 37 (15.55) & 35 (14.71) & 35 (14.71) & $25(10.5)$ & \\
\hline \multicolumn{7}{|l|}{ Marital Status } \\
\hline Single & $6(2.52)$ & $28(11.76)$ & $27(11.34)$ & $32(13.45)$ & $18(7.56)$ & 0.326 \\
\hline Married & $16(6.72)$ & $28(11.76)$ & $24(10.08)$ & $17(7.14)$ & $22(9.24)$ & \\
\hline Divorced & $2(0.84)$ & $2(0.84)$ & $5(2.1)$ & $3(1.26)$ & $5(2.1)$ & \\
\hline Widowed & $0(0)$ & $1(0.42)$ & $1(0.42)$ & $1(0.42)$ & $0(0)$ & \\
\hline \multicolumn{7}{|l|}{ Residency } \\
\hline Urban & $21(8.82)$ & $51(21.43)$ & 46 (19.33) & $40(16.81)$ & 36 (15.13) & 0.575 \\
\hline Rural & $3(1.26)$ & $8(3.36)$ & $11(4.62)$ & $13(5.46)$ & $9(3.78)$ & \\
\hline \multicolumn{7}{|l|}{ Education } \\
\hline Primary school & $0(0)$ & $0(0)$ & $1(0.42)$ & $0(0)$ & $1(0.42)$ & 0.005 \\
\hline Intermediate school & $1(0.42)$ & $0(0)$ & $0(0)$ & $0(0)$ & $5(2.1)$ & \\
\hline Secondary School & $2(0.84)$ & $15(6.3)$ & $20(8.4)$ & $14(5.88)$ & $10(4.2)$ & \\
\hline University or college & $16(6.72)$ & $40(16.81)$ & $29(12.18)$ & 37 (15.55) & $25(10.5)$ & \\
\hline Postgraduate & $5(2.1)$ & $4(1.68)$ & $7(2.94)$ & $2(0.84)$ & $4(1.68)$ & \\
\hline \multicolumn{7}{|l|}{ Household Income } \\
\hline Less than 5000 Saudi Riyal & $4(1.68)$ & $11(4.62)$ & $12(5.04)$ & $18(7.56)$ & $9(3.78)$ & 0.427 \\
\hline 5000 - 10000 Saudi Riyal & $6(2.52)$ & $11(4.62)$ & $8(3.36)$ & $13(5.46)$ & $9(3.78)$ & \\
\hline 10001 - 15000 Saudi Riyal & $5(2.1)$ & $15(6.3)$ & $12(5.04)$ & $7(2.94)$ & $13(5.46)$ & \\
\hline 15001 - 20000 Saudi Riyal & $4(1.68)$ & $9(3.78)$ & $14(5.88)$ & $6(2.52)$ & $2(0.84)$ & \\
\hline 20001 - 25000 Saudi Riyal & $2(0.84)$ & $3(1.26)$ & $2(0.84)$ & $4(1.68)$ & $5(2.1)$ & \\
\hline More than 25000 Saudi Riyal & $3(1.26)$ & $10(4.2)$ & $9(3.78)$ & $5(2.1)$ & $7(2.94)$ & \\
\hline \multicolumn{7}{|l|}{ Employment Status } \\
\hline Employed & $16(6.72)$ & $29(12.18)$ & $20(8.4)$ & $18(7.56)$ & $18(7.56)$ & 0.133 \\
\hline Unemployed/housewife & $6(2.52)$ & $22(9.24)$ & 28 (11.76) & $23(9.66)$ & $16(6.72)$ & \\
\hline Student & $2(0.84)$ & $8(3.36)$ & $9(3.78)$ & $12(5.04)$ & $11(4.62)$ & \\
\hline \multicolumn{7}{|l|}{ Smoking Status } \\
\hline Heavy smoker & $2(0.84)$ & $6(2.52)$ & $5(2.1)$ & $14(5.88)$ & $10(4.2)$ & 0.031 \\
\hline Social smoker & $0(0)$ & $10(4.2)$ & $5(2.1)$ & $4(1.68)$ & $5(2.1)$ & \\
\hline Not a smoker & $22(9.24)$ & $43(18.07)$ & 47 (19.75) & $35(14.71)$ & $30(12.61)$ & \\
\hline
\end{tabular}

TABLE 5: Depression severity associated with sociodemographic factors

Note: Based on Chi-Square test of independence. 
The medical care has a lot to be reviewed and improved within the framework of the association between chronic diseases and psychiatric disorders such as depression. The development of psychiatric conditions is still an area of research and remains unknown, giving the opportunity for several interpretations to rise. Some early studies have shown that radiologically hyperintense lesions in prefrontal and temporal cortical regions lead to structural changes responsible for depression among MS patients [24]. Others have found that lesions in cortical and subcortical projections are responsible for the pathogenesis of depression [25]. We aimed to assess the point prevalence of depression and its severity within a sample of MS patients in Saudi Arabia. Our approach differs from others in the literature since it operates a modified screening tool dedicated to the illness severity. In addition, the prevalence of depression in our model of MS patients was high probably because of maltreatment or failure of pharmacological and non-pharmacological means during the management plan. Our study findings indicated that the point prevalence of depression in MS patients was (89.9\%), which is higher than in Norway (31.4\%), Canada (34.4\%), the Islamic Republic of Iran (59.4\%), as well as the reported number of the general population in the geographical region (6.6\%) $[15,17,18,20]$. In the current study, high frequency of MS patients with mild-moderate depression was found, which is consistent with the results of the previous studies [20,26]. Moreover, the frequency of severe level of depression in our study was $18.9 \%$. Similar results were also found in Iran where they reported that $18.1 \%$ of MS patients had severe depression [20]. The little variation could be attributed to the demographic and environmental similarities between the two countries. On the other hand, the prevalence of severe depression is low when compared to Tunisian MS patients which was 25\% [19]. Our results showed differences in terms of gender discrepancy since the female MS patients reported more vulnerability to manifest depression symptoms compared to males. The findings are compatible with the Iranian study and marginally consistent with the Norway study [17,20]. However, our data does not have a statistically supported assumption despite the unique sociodemographic morphology and gender role the Saudi women play when compared to Western communities. We noted that there were no variations in the frequency of reporting depressive symptoms regardless of the current state of marital status. Similarly, patients who are currently unemployed or housewives had a higher frequency of reporting depressive symptoms compared to students or employed patients. Furthermore, there were significant differences in the frequency of reporting depressive symptoms and patients' smoking status. However, our findings are inconsistent with recent publications $[27,28]$. Lastly, our results show that higher level of education had a positive correlation with depression severities, which contradicts existing literature [20].

\section{Study limitations}

The results of the present study need to be evaluated carefully since we did not address the issue of social support and side effects of MS medications. Moreover, the cross-sectional nature of our data does not establish cause and effect relationship between the variables. The study was quasi population-based with a relatively small sample. In addition, $\mathrm{PHQ}-9$ does not screen for other psychiatric comorbidities that may have a similar impact such as anxiety.

\section{Conclusions}

High levels of depression symptoms were recorded among MS patients in Saudi Arabia. The relationship between MS and psychiatric conditions does exist despite the uncertainty of its pathogenesis. There is a need for further longitudinal studies and clinical trials to validate the impact of depression on MS patients in Saudi Arabia. The MS treating neurologists should actively investigate depressive symptoms at the time of diagnosis and throughout the disease management course to provide vital data.

\section{Additional Information \\ Disclosures}

Human subjects: Consent was obtained by all participants in this study. Animal subjects: All authors have confirmed that this study did not involve animal subjects or tissue. Conflicts of interest: In compliance with the ICMJE uniform disclosure form, all authors declare the following: Payment/services info: All authors have declared that no financial support was received from any organization for the submitted work. Financial relationships: All authors have declared that they have no financial relationships at present or within the previous three years with any organizations that might have an interest in the submitted work. Other relationships: All authors have declared that there are no other relationships or activities that could appear to have influenced the submitted work.

\section{Acknowledgements}

Special thanks to Department of Psychiatry, College of Medicine, King Saud University and SABIC Psychological Health Research \& Applications Chair (SPHRAC) for helping us with the Arabic version of PHQ scale. We would also like to thank all the patients who participated in the study.

\section{References}

1. Vos T, Allen C, Arora M, et al.: Global, regional, and national incidence, prevalence, and years lived with disability for 310 diseases and injuries, 1990-2015: a systematic analysis for the Global Burden of Disease Study 2015. Lancet. 2016, 388:1545-1602. 10.1016/S0140-6736(16)31678-6 
2. Vos T, Abajobir AA, Abate KH, et al.: Global, regional, and national incidence, prevalence, and years lived with disability for 328 diseases and injuries for 195 countries, 1990-2016: a systematic analysis for the Global Burden of Disease Study 2016. Lancet. 2017, 390:1211-1259. 10.1016/S0140-6736(17)32154-2

3. Garg N, Smith TW: An update on immunopathogenesis, diagnosis, and treatment of multiple sclerosis . Brain Behav. 2015, 5:00362. 10.1002/brb3.362

4. Comabella M, Khoury SJ: Immunopathogenesis of multiple sclerosis. Clin Immunol. 2012, 142:2-8. 10.1016/j.clim.2011.03.004

5. Selter RC, Hemmer B: Update on immunopathogenesis and immunotherapy in multiple sclerosis . ImmunoTargets Ther. 2013, 2:21-30. 10.2147/ITT.S31813

6. Poorolajal J, Bahrami M, Karami M, Hooshmand E: Effect of smoking on multiple sclerosis: a meta-analysis . J Public Health. 2017, 39:312-320. 10.1093/pubmed/fdw030

7. Degelman ML, Herman KM: Smoking and multiple sclerosis: a systematic review and meta-analysis using the Bradford Hill criteria for causation. Mult Scler Relat Disord. 2017, 17:207-216. 10.1016/j.msard.2017.07.020

8. World Health Organization \& Multiple Sclerosis International Federation: Atlas: Multiple Sclerosis Resources in the World 2008. WHO, 2008.

9. American Psychiatric Association: Diagnostic and Statistical Manual of Mental Disorders (DSM-5 ${ }^{\circledR}$ ) . American Psychiatric Association, 2013. 10.1176/appi.books. 9780890425596

10. Mayne RG: An Expository Lexicon of the Terms, Ancient and Modern, in Medical and General Science: Including a Complete Medico-legal Vocabulary, and Presenting the Correct Pronunciation, Derivation... of the Names, Analogues... Connected with Medicine and Employed in the Natural Sciences. John Churchill, London; 1860.

11. Molendijk ML, Spinhoven P, Polak M, Bus BA, Penninx BW, Elzinga BM: Serum BDNF concentrations as peripheral manifestations of depression: evidence from a systematic review and meta-analyses on 179 associations ( $\mathrm{N}$ = 9484). Mol Psychiatry. 2014, 19:791-800. 10.1038/mp.2013.105

12. Ruhé HG, Mason NS, Schene AH: Mood is indirectly related to serotonin, norepinephrine and dopamine levels in humans: a meta-analysis of monoamine depletion studies. Mol Psychiatry. 2007, 12:331-359. 10.1038/sj.mp.4001949

13. Weissman MM, Bland RC, Canino GJ, et al.: Cross-national epidemiology of major depression and bipolar disorder. JAMA. 1996, 276:293-299. 10.1001/jama.1996.03540040037030

14. Eaton WW, Anthony JC, Gallo J, et al.: Natural history of Diagnostic Interview Schedule/DSM-IV major depression: the Baltimore epidemiologic catchment area follow-up. Arch Gen Psychiatry. 1997, 54:993-999. 10.1001/archpsyc.1997.01830230023003

15. Ferrari AJ, Somerville AJ, Baxter AJ, Norman R, Patten SB, Vos T, Whiteford HA: Global variation in the prevalence and incidence of major depressive disorder: a systematic review of the epidemiological literature. Psychol Med. 2013, 43:471-481. 10.1017/S0033291712001511

16. World Federation for Mental Health: Depression: A Global Crisis. World Mental Health Day, October 10 2012. World Federation for Mental Health, Occoquan; 2012.

17. Beiske AG, Svensson E, Sandanger I, Czujko B, Pedersen ED, Aarseth JH, Myhr KM: Depression and anxiety amongst multiple sclerosis patients. EurJ Neurol. 2008, 15:239-245. 10.1111/j.1468-1331.2007.02041.x

18. Sadovnick AD, Remick RA, Allen J, et al.: Depression and multiple sclerosis . Neurology. 1996, 46:628-632. 10.1212/WNL.46.3.628

19. Minden SL, Orav J, Reich P: Depression in multiple sclerosis . Gen Hosp Psychiatry. 1987, 9:426-434. 10.1016/0163-8343(87)90052-1

20. Saadat SM, Hosseininezhad M, Bakhshayesh B, Saadat SN, Nabizadeh SP: Prevalence and predictors of depression in Iranian patients with multiple sclerosis: a population-based study. Neurol Sci. 2014, 35:735740. 10.1007/s10072-013-1593-4

21. Goodman LA: Snowball sampling. Ann Math Stat. 1961, 32:148-170.

22. AlHadi AN, AlAteeq DA, Al-Sharif E, et al.: An Arabic translation, reliability, and validation of Patient Health Questionnaire in a Saudi sample. Ann Gen Psychiatry. 2017, 16:32. 10.1186/s12991-017-0155-1

23. Kroenke K, Spitzer RL, Williams JB: The PHQ-9: validity of a brief depression severity measure . J Gen Intern Med. 2001, 16:606-613. 10.1046/j.1525-1497.2001.016009606.x

24. Feinstein A, Roy P, Lobaugh N, Feinstein K, O’connor P, Black S: Structural brain abnormalities in multiple sclerosis patients with major depression. Neurology. 2004, 62:586-590. 10.1212/01.WNL.0000110316.12086.0C

25. Berg D, Supprian T, Thomae J, et al.: Lesion pattern in patients with multiple sclerosis and depression . Mult Scler J. 2000, 6:156-162. 10.1177/135245850000600304

26. Gay MC, Vrignaud P, Garitte C, Meunier C: Predictors of depression in multiple sclerosis patients . Acta Neurol Scand. 2010, 121:161-170. 10.1111/j.1600-0404.2009.01232.x

27. Newland P, Flick L, Salter A, Dixon D, Jensen MP: The link between smoking status and co-morbid conditions in individuals with multiple sclerosis (MS). Disabil Health J. 2017, 10:587-591. 10.1016/j.dhjo.2017.03.005

28. Taylor KL, Hadgkiss EJ, Jelinek GA, Weiland TJ, Pereira NG, Marck CH, van der Meer DM: Lifestyle factors, demographics and medications associated with depression risk in an international sample of people with multiple sclerosis. BMC Psychiatry. 2014, 14:327. 10.1186/s12888-014-0327-3 